\title{
Author Correction: Data visualisation to support obesity policy: case studies of data tools for planning and transport policy in the UK
}

\author{
Pablo Monsivais $^{1,2} \cdot$ Oliver Francis $^{1} \cdot$ Robin Lovelace $^{3} \cdot$ Michael Chang $^{4,5} \cdot$ Emma Strachan $^{6} \cdot$ Thomas Burgoine $^{1}$
}

Published online: 15 February 2019

(c) Springer Nature Limited 2019

Correction to: International Journal of Obesity 42: 1977-1986; https://doi.org/10.1038/s41366-018-0243-6; published online 23 November 2018

In the original version of this Article the following funding details were omitted from the Acknowledgements section:

"This work was undertaken by the Centre for Diet and Activity Research (CEDAR), a UK Clinical Research Collaboration (UKCRC) Public Health Research Centre of
Excellence. Funding from the British Heart Foundation, Cancer Research UK, Economic and Social Research Council, Medical Research Council, the National Institute for Health Research [grant number ES/G007462/1], and the Wellcome Trust [grant number 087636/Z/08/Z], under the auspices of the UK Clinical Research Collaboration, is gratefully acknowledged"

The authors apologise for any inconvenience caused.

This has now been corrected in both the PDF and HTML versions of the Article.

Pablo Monsivais

p.monsivais@wsu.edu

1 UKCRC Centre for Diet and Activity Research (CEDAR), MRC Epidemiology Unit, University of Cambridge School of Clinical Medicine, Institute of Metabolic Science, Cambridge Biomedical Campus, Cambridge CB2 0QQ, UK

2 Department of Nutrition and Exercise Physiology, Elson S. Floyd College of Medicine, Washington State University, Spokane, WA 99210, USA

3 Institute for Transport Studies, University of Leeds, Leeds LS2 9JT, UK

4 Town \& Country Planning Association, London SW1Y 5AS, UK

5 Carnegie School of Sport, Leeds Beckett University, Leeds LS6 3QT, UK

6 Health Improvement, Leeds City Council, Leeds LS2 9JT, UK 